\title{
Author Index Vol. 2,1995
}

(A) $=$ Abstract

Ackenheil,M. 115 Ader.R. 357(A) Agrasal, C. 54 Aguila,M.C 166 AIperina,E. 364(A)

Bakker,J.M. 358(A) Bancroft, G.J. 361(A) Banks, W.A. 161,241 Bany,U. 25 Bartlett,D. 365(A) Benigni, F. 2 Ben-Yosef, D. 249 Berczi.I. 184 Blatteis,C.M. 282 Blom,J.M.C. 92 Bonavida, B. 16 Bongiorno, P.B. 141 Braczkowski, R. 361 (A) Brenan,M. 362(A) BroadweU, R.D. 241

Call.G.B. 299

Carpenter, G.W. 44 Carr,DJ.J. 44 Catania, A. 258 Chambers, C. 365 (A) Chandler, S. 365(A) Clarke, G. 363(A) Clements, J.M. 365(A) Cohen, N. 357(A) Conde,G.L. 363(A) Conn.CA. 216 Corkill.D. 365(A) Corkill.H. 365(A) Coward, L. 365(A) Crimmin.M. 365(A)

Dantzer.R. 357(A) Dardenne,M. 313 Davidson, R. 359(A) Davis, M.H. 365(A) Delrue-Perollet, C. 36 Delvaux,A. 149 Devonio.L. 358(A) Ding, G.F. 82, 274 Di Santo, E. 149 Dunn, A J. 319

Egleton,R. 365(A) Esquiilno, A.I. 54

Faggioni, R. 2 Fan.S.G. 82,274 Fontani.G. 155 Franey,S. 365(A) Frenzel, K.-H. 115 Fujiwara, R, 174 
Gan,X.-H. 16

Gao,S. 274 Gearing, A.J.H. 365(A) Geenen.V. 360(A) Ghezzi, P. 2,149 Giordano, M. 54 Girolami, L. 155 Gnocchi,P. 149 Gold,P.W. 141

Goldman, M. 149 Gorospe,W.C. 299 Grossman, A. 356 Grzeszczak, W.I. 361(A) Guida,L. 361(A)

Hampel,H. 115

Harbuz, M, 356, 360 (A), 363 (A) Hare,A.S. 363(A) Hart,R.P. 236,347 Hawrylowicz, CM. 361(A) Heidmann, D. 115 Heijnen.CJ. 364 (A), 365 (A)

Helfrich,K. 365(A) Hermann, G. 74 Hernandez-Pando.R. 359(A) Holtsclaw, L.I. 329 Honour, J. 359(A) Hori.T. 203

Idova,G. 358(A) Isetta,AM. 149

Jessop.D. 360(A) Jonakait, G.M. 236,347 Judd,A.M. 299

Kageyama, K. 137 Karanth,S. 166 Karapetrovic, B. 100 Karp.J.D. 357(A) Kastin,A.J. 161,241 Katafuchi.T. 203 Kavelaars.A. 364 (A), 365 (A)

Kluger, M.J. 216 Komori, T. 174 Kon,O.M. 359(A) Kosec.D. 100 Kozak,W. 216 Kretzschmar, H.A. 115 Kroes,H. 358(A) Kruszewska, B. 357(A)

Kuchinke.W. 347 Kuis,W. 365(A) Kusnecov, A. 364 (A)

Larsen,P. 360(A) Laskowska-Bozek, H. 25 Leon,L.R. 216 Leonard, B.E. 263 Leposavic, G. 100 
Levine,J.E. 290

Levitan, G. 249

Li,K.-S. 36

Li,Y.F. 82,274

Licinio,J. 141

Lightman, S. 357 (A), 360 (A), 363 (A)

Lipton.J.M. 1,181,258

Lupo.C. 155

LuryJ. 365(A)

Lyson, K. 166

McCann,S.M. 1,166,181 Mangan.M.H. 365(A) Marchant,A. 149 Martens, H. 360(A) Massimini, N. 31 Matyzak,M. 362 (A), 365 (A) Mei,L. 82,274 Mello-Coelho, V. de 313 Micic,M. 100 Miller, K.M. 365(A) Miller, S.C. 329 Moreno, M.L. 54 Moser, D.K. 16 Moynihan.J.A. 357(A) Miiller.N. 115 Murphy, D. 360(A) Muscettola, M. 155

Needham,M. 362(A) Nelson, L. 364(A) Nelson, RJ. 92 Neveu, P.J. 36 Niijima,A. 203 Nobel, C.S.I. 61 Nomura, J. 174

OIadehin,A. 282 Ottaway.CA. 358(A) OvadiaJL 249,339

Panerai.A.E. 359(A) Parrott,R.F. 359(A) Parry, D. 365(A) Pavina.T. 364(A) Penning, R. 115 Perry, V.H. 362 (A), 365 (A) Phillips, M.J. 362(A) Plioplys, A.V. 31 Plotkin,S.R. 161 PozzLP. 149 Prous.D. 249 Prystowsky, M.B. 290

Rapier, S.H. 329

Renshaw, D. 360 (A), 363 \$Q

Rivier.C. 224 Robert, F. 360(A) Rogers, R.C. 74 Rohn,W.M. 108 
Rollinson, C. 365(A) Romanowski, W. 361(A) Rook, GA.W. 359 (A) Rouppe vd Voort, C. 365 (A) Ry4ewski,J. 25

Sabbadini,E. 184 Sacerdote,P. 359(A) Sasaki, S. 88,134 Savino.W. 313 Schmidt, ED. 358(A) Schultzberg, M. 61 Sei,Y. 121 Shanks, N. 364(A) Shao, L. 274 Shaw, RJ. 359 (A) Shiber.J.R. 92 Shimizu,N. 203 Sigola,L. 361(A) Sironi,M. 149 Smith, T. 356 Sobcho,A. 339 Song,C. 263 
Soszynski, D. 216 Spangelo, B.L. 299 Spector.N.H. 182 Stabler, G. 365 (A)

Stokarska, G. 25 Suffredini, A.F. 258

Take,S. 203 Takebe,K. 88,134,137 Tamarkin,L. 92 Tanganelli, C. 155 Tanida.M. 174 Tarcic,N. 249 Tildere, FJ.H. 358(A) Tolchard.S. 363(A) TumbuIl.A. 224 Turner, B.B. 329

VandePolM 364(A) VanderVoort.Ch.R. 364(A) VanRees.E.P. 358(A) Vellucci, SV. 359(A) Villanua, MA. 54 
Vitkovia,L. 121 Vredevoe, D.L. 16

Wang, J. 319 Ward,GA. 365(A) Warwick-Evans, LA. 365 (A) Watanobe.H. 88,134,137 Watkins.A. 356 Weidenfeld, J. 339 Weigent,DA. 108 Weiss, D.W. 249 Weller,R.O. 362(A) Wells, G. 365(A) Wholmann.A. 339 Wilkinson, RJ. 359(A) Wong,M.-L. 141 Wood,M. 365(A)

Yokoyama, M.M. 121,174

Zubelewicz, B. 361(A) Zumwalt,J. 299 Zuo.Y.C. 82

Author Index Vjj, 1995 